\title{
UPLC-MS/MS characterization of phenolic constituents in fennel seed extracts (Foeniculum vulgare Mill.)
}

\section{Abstract}

Reports in the recent literature indicate that consumption of phytochemicals (e.g. plant polyphenols) has been associated with a wide range of health benefits. Fennel (Foeniculum vulgare Mill.) is common Mediterranean plant that has been widely used as medicinal and aromatic herb. Numerous studies reported the use of fennel extracts (plant or seed) due to its diversity of phenolic compounds to whom inhibitory effects against several diseases are prescribed. Hence, phenolic compounds isolated from fennel are of the great interest for the use in food, chemical and pharmaceutical industries.

In order to obtain the highest quality of the fennel extracts, the selection of an effective extraction procedure is required. Nowadays, innovative extraction techniques have been introduced for isolation of bioactive compounds from plant material, among which pressurized liquid extraction (PLE) was reported as automated extraction procedure that combines effects of elevated temperature and pressure to increase the efficiency of the extraction process. Improved efficiency is also achieved by selecting the right solvent and by optimizing processing conditions (temperature, static time, cycle number).

Thus, PLE was applied for the extraction of phenolic compounds from fennel seeds. The effects of exhaustive extraction (acetone followed by methanol) as well as extraction temperatures $\left(75\right.$ and $\left.100{ }^{\circ} \mathrm{C}\right)$, static time $(5$ and $10 \mathrm{~min})$ and cycle number $(1,2,3)$ on the phenolic compounds concentrations were evaluated. A total of 27 phenolic compounds were identified and characterized by UPLC-ESI-MS/MS whereas 6 different categories are distinguished: (1) simple phenolic acid derivatives, (2) hydroxycinnamoylquinic acid derivatives, (3) flavonoid diglycosides, (4) flavonoid $O$-rhamnoglucosides, (5) flavonoid glucuronides and (6) flavonoid glycosides. Phenolic compounds were identified by comparing retention time and $\mathrm{m} / \mathrm{z}$ values obtained by MS and MS/MS with the mass spectra from corresponding standards whereas the quantification was made on the basis of their peak areas and comparison with calibration curve obtained with the corresponding standards. Obtained results indicated that hydroxycinnamoylquinic acid derivatives (10 compounds) and flavonoid glucuronides (3 compounds) were found as the most abundant phenolics in acetone and methanol extracts, respectively. Statistical analysis showed that optimized PLE conditions for the most effective extraction of phenolic compounds were as follows: $100{ }^{\circ} \mathrm{C} / 10 \mathrm{~min} / 3$ cycles for acetone and $100{ }^{\circ} \mathrm{C} / 5 \mathrm{~min} / 3$ cycles for methanol extracts. In conclusion, PLE might be considered as a rapid and effective extraction procedure that could be useful in production of fennel seeds extracts.

\section{Conflict of Interest}

There is no conflict of interest. 